\title{
Stimulated Emission Depletion and Fluorescence Correlation Spectroscopy of a Branched Quadrupolar Chromophore.
}

\author{
D. A. Armoogum ${ }^{1}$, R. J. Marsh ${ }^{1}$, N. Nicolaou ${ }^{1}$, O. Mongin ${ }^{2}$, M. Blanchard-Desce ${ }^{2}$, and A. J. Bain ${ }^{1}$ * \\ 1. Department of Physics \& Astronomy, University College London, Gower Street, London WC1E \\ 6BT \\ 2. Synthèse et ElectroSynthèse Organiques, (CNRS, UMR 6510), Université de Rennes 1, Campus \\ de \\ Beaulieu, Bât 10A Case 1003, F-35042 Rennes Cedex, France
}

\begin{abstract}
Stimulated emission depletion (STED) and single molecule fluorescence correlation spectroscopy (FCS) are used to determine stimulated emission cross-sections and investigate non-radiative relaxation in a branched quadrupolar chromophore (OM77). The results are used as inputs to simulations of single molecule STED by which the feasibility of STED control of the single molecule fluorescence cycle can be assessed. Single molecule STED in OM77 is shown to be readily achievable; however its effectiveness in reducing triplet trapping is apparently mediated by fast non-radiative relaxation processes other than intersystem crossing and rapid quenching of the triplet state in a non-deoxygenated environment.
\end{abstract}

\section{KEYWORDS}

Stimulated Emission Depletion, Fluorescence Correlation Spectroscopy, Single Molecule, Non-radiative relaxation.

\section{BACKGROUND AND CONTEXT}

Single molecule fluorescence has widespread application across the life sciences ${ }^{1-6}$ but is subject to the random nature of spontaneous relaxation pathways between the excited states of a single molecule. Processes that give rise to substantial (microsecond-millisecond) residence times in so called 'dark states', where the molecule is unable to absorb and emit radiation $^{4}$, are significant complications to single molecule fluorescence experiments and can lead to the misinterpretation of data. Intersystem crossing (spin conversion) to the triplet manifold is one such mechanism with the forbidden nature of spontaneous emission between different spin states delaying return to the ground state and subsequent re-excitation.

The ability to exert control on the absorption emission cycle in single molecule fluorescence represents a fundamental step change with the potential to significantly enhance the accuracy and precision of single molecule spectroscopy and microscopy. The reduction in false error rates in DNA sequencing ${ }^{5}$ and increased sensitivity in high throughput drug screening would be of significant benefit to both the Biotechnology and Pharmaceutical industries. Additionally the increased precision ${ }^{7}$ afforded to single molecule fluorescence techniques such as FRET (fluorescence/Förster resonance energy transfer) and the development of new techniques in single molecule time resolved spectroscopy would be of significant benefit to research in the life sciences and nanotechnology.

\footnotetext{
*Corresponding Author, email: a.bain@ucl.ac.uk
} 
Preliminary theoretical modelling by $\mathrm{us}^{8}$ had indicated the possibility of reducing transitions to the triplet state and increasing the fluorescence signal using STED. The model is based on a stochastic simulation of the single molecule fluorescence cycle for mode-locked excitation using the probabilities of excitation, intersystem crossing, fluorescence emission and triplet relaxation as inputs. STED is introduced into the simulation by the addition of a finite DUMP probability at a set (controllable) delay after the excitation (PUMP) pulse. This paper details a feasibility study of single molecule STED control of the fluorescence cycle, by the experimental measurement of STED characteristics and photophysical parameters via FCS of a branched quadrupolar chromophore and computer simulations to determine optimal conditions for STED control of the single molecule fluorescence cycle.

\section{MATERIALS AND METHODS I - STED}

\subsection{Identification and characterisation of chromophores for single molecule STED}

The branched fluorescent probe (OM77) used in this study is shown in figure 1, designed for optimal near infra-red twophoton absorption and single photon fluorescence yield ${ }^{9}$. It has a substantial two-photon cross-section and a fast ground state vibrational relaxation time ${ }^{10}$ which makes it an ideal candidate for single molecule STED ${ }^{8}$. The quantum yield of the fluorophore is $0.74^{9}$ indicating significant non-radiative de-excitation in the excited singlet $\left(\mathrm{S}_{1}\right)$ state.

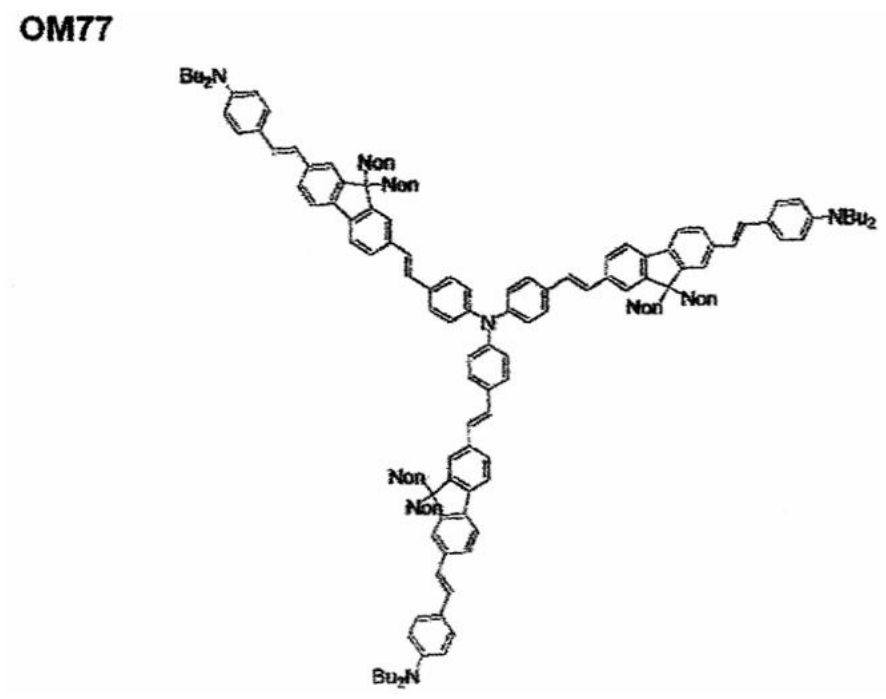

Figure 1. Structure of the branched quadrupolar fluorophore OM77 used in this study. OM77 consists of a branched (planar) arrangement of three fluorene-based quadrupolar units.

\subsection{Experimental apparatus used for bulk STED measurements}

Preliminary models indicated that a very high depletion probability is necessary to enhance single molecule fluorescence signals using STED $^{8}$. In order to check the feasibility of high probability depletion, STED measurements were made in bulk sample with the aim of achieving a very large fractional population removal from the excited state $(>90 \%$ depletion). The procedure for this experiment has been described in detail elsewhere ${ }^{10,11}$; the apparatus used for these measurements is shown in figure 2 . 


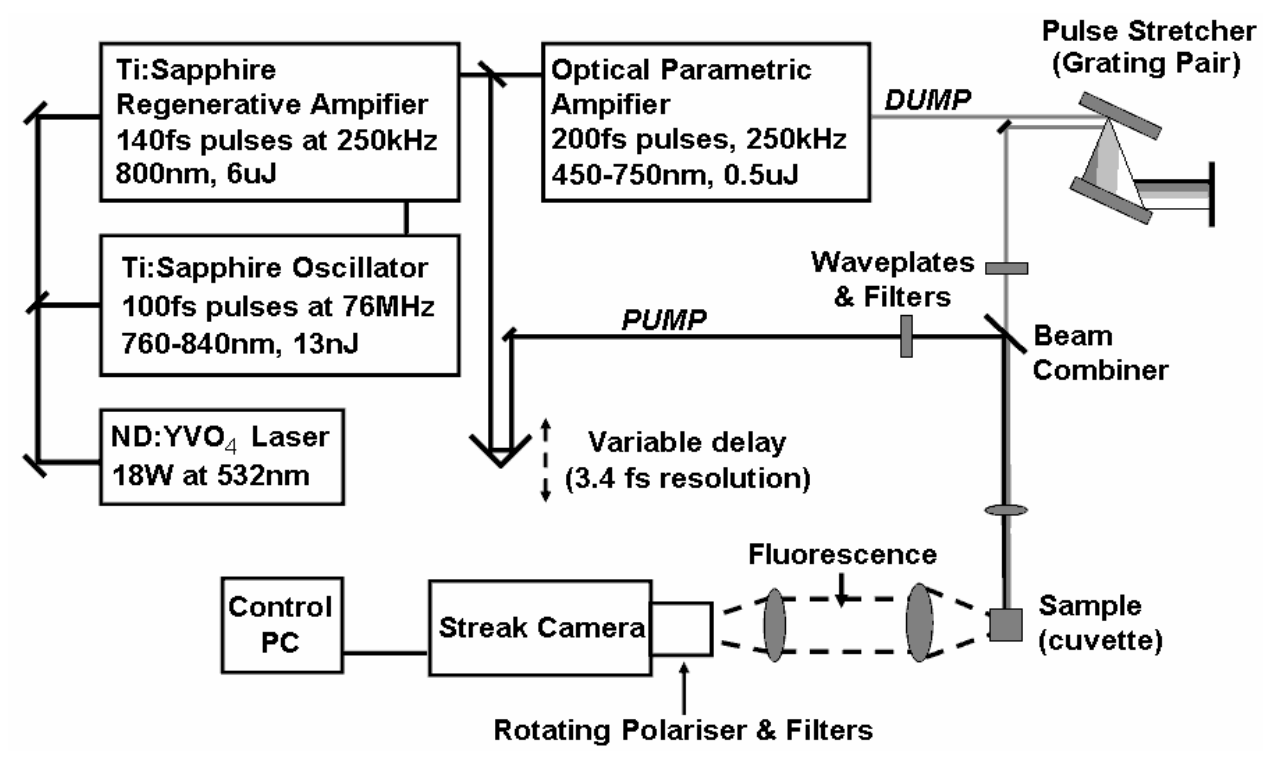

Figure 2. Experimental apparatus used for the study of STED population depletion dynamics.

Following two-photon excitation (PUMP) from the partial output of an infra-red regenerative amplifier (Coherent RegA 9000), a fraction of the excited state population is stimulated back to the ground state via visible radiation (DUMP) from an Optical Parametric Amplifier (OPA, Coherent 9400). Under normal operating conditions the OPA output pulse width is ca. 200fs - using pulses this short would result in significant re-pumping of the excited state from un-relaxed vibrational levels in the ground state that are initially populated as a result of stimulated emission, thereby reducing STED efficiency. Furthermore, OM77 is an efficient two-photon absorbing system in the visible where we have recently identified the onset of a giant two-photon resonance (ca. $12000 \mathrm{GM}$ at $570 \mathrm{~nm})^{9}$ and the probability of DUMP excited fluorescence from ground state molecules needs to be minimized. To limit these effects, the DUMP pulses were temporally stretched using a grating pair to greater than 20 picoseconds.

\section{STED RESULTS AND SIMULATIONS}

\subsection{STED Results}

Excited state depletion for OM77 was measured as a function of DUMP energy and wavelength. The variation in fractional population removal for a DUMP wavelength of $551 \mathrm{~nm}$ is shown in figure 3a. STED experiments in molecular populations are limited to wavelengths at which the (necessarily) large flux of DUMP photons does not significantly excite un-pumped ground state molecules. In single molecule STED the situation is different; overlap between the DUMP pulse and the absorption spectrum of the molecule would lead to significant probability of re-pumping into the excited state for any molecules spontaneously relaxing in the time window between the PUMP and DUMP pulses. This would count as excitation without STED leading to longer excited state residence times and an increased probability of triplet trapping. To determine the conditions necessary for efficient single molecule STED fluorescence depletion measurements as a function of DUMP pulse energy at varying wavelengths were recorded using a streak camera (Hamamatsu C4334). Population depletion curves at DUMP wavelengths up to the onset of measurable DUMP induced fluorescence were analysed as in previous studies ${ }^{12}$ to yield STED cross-sections whose wavelength dependence was found to correlate well with the fluorescence spectrum (figure 3b). The maximum STED cross-sections prior to the onset of DUMP induced fluorescence are displayed in table 1 together with the saturation parameter S required for a strong DUMP probability (indicated in parentheses). The DUMP energy required for an equivalent transition probability in a single molecule experiment can then be calculated. OM77 requires DUMP energies in the picojoule range, 
corresponding to on-sample average powers (at $76 \mathrm{MHz}$ ) of ca. $200 \mu \mathrm{W}$. This performance can easily be met by synchronously pumped Optical Parametric Oscillators and amplified diode laser systems.
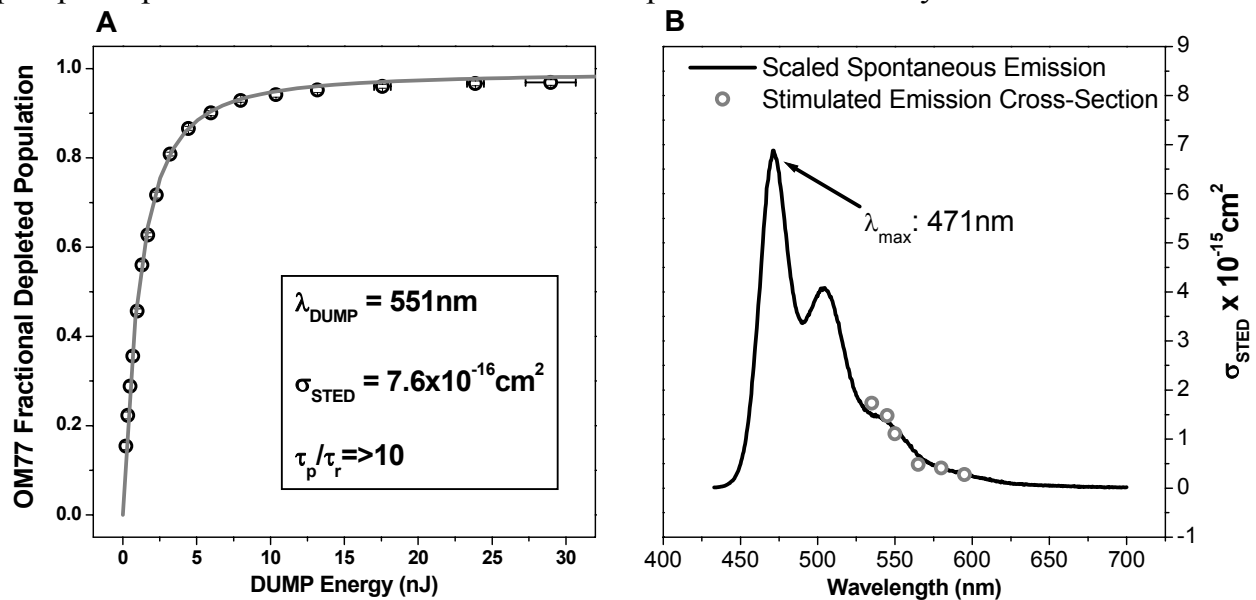

Figure 3: (A) STED fractional population removal as a function of DUMP energy in OM77, showing depletion levels in excess of $95 \%$. (B) Measured relative values of the stimulated emission cross-section in OM77 are in good agreement with the spontaneous emission spectrum, indicating that the efficiency of single molecule STED should reach a maximum for a DUMP wavelength of $471 \mathrm{~nm}$.

\begin{tabular}{|l|c|c|c|c|}
\hline & $\lambda_{\text {MAX }}$ & $\sigma_{\text {STED }} \times 10^{-15} \mathrm{~cm}^{2}$ & $S=\frac{\sigma_{\text {STED }} E_{\text {DUMP }}}{A h v}$ & E $_{\text {DUMP }}$ Confocal $\left(\times 10^{-12} \mathrm{~J}\right)$ \\
\hline OM77 & $471 \mathrm{~nm}$ & 6.88 & $21(96 \%)$ & 2.58 \\
\hline
\end{tabular}

Table 1. The maximum STED cross-section attainable for a single OM77 molecule without significant re-pumping, the saturation parameter $S$ required for strong depletion (DUMP probability) and the corresponding on-sample DUMP energy required in a confocal microscope arrangement $\left(\mathrm{A} \cong 2 \times 10^{-9} \mathrm{~cm}^{2}\right)$.

\subsection{STED Simulations}

Stochastic simulations of the effects of STED on single molecule fluorescence were performed for a range of radiative and non-radiative decay rates. The Jablonski diagram in figure 4 corresponds to a molecule with a quantum yield of 0.9 , a fluorescence lifetime of $1 \mathrm{~ns}$ and a triplet recovery time of 8 microseconds.

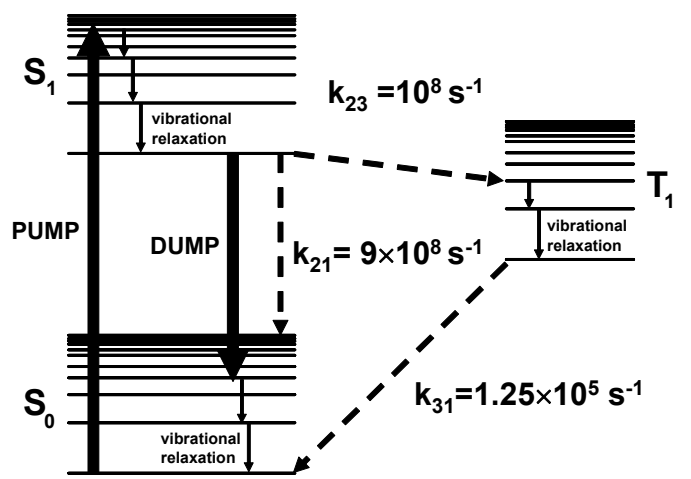

Figure 4. Jablonski diagram showing typical relaxation pathways open to an excited fluorophore. Vibrational relaxation is rapid (subpicosecond ${ }^{10}$ ) and not included in the simulations. 
Results of simulations using these parameters with a DUMP probability of 0.95 (cf. OM77) varying excitation probability and PUMP-DUMP delay are shown in figures 5a and 5b. From figure $5 \mathrm{a}$ it can be seen that STED enhancement of fluorescence increases with excitation probability at short PUMP-DUMP delays and is independent thereafter. From figure $5 \mathrm{~b}$ shows that a PUMP-DUMP delay of approximately half the $\mathrm{S}_{1}$ lifetime yields the greatest signal enhancement over a range of excitation probabilities. STED enhancement critically depends on the triplet recovery time as shown in figure $5 \mathrm{c}$, where the fluorescence enhancement increases with triplet recovery time reaching a maximum value at ca. $10^{5} \tau_{\mathrm{F}}\left(\tau_{\mathrm{F}}=1 \mathrm{~ns}\right)$.
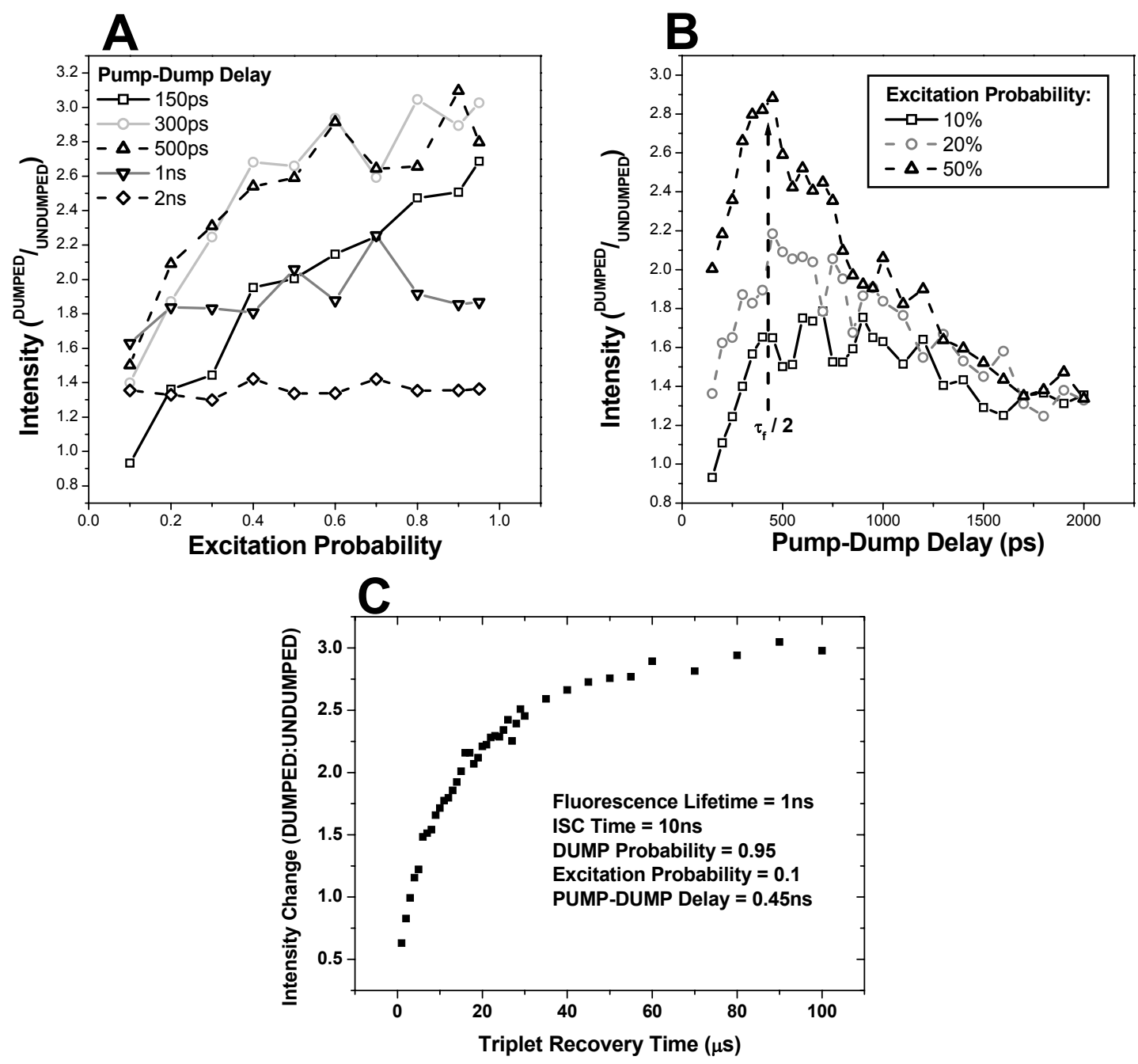

Figure 5: (A) The effect of excitation probability on the enhancement of fluorescence as a function of PUMP-DUMP delay (DUMP probability 0.95). (B) Variation in the DUMPED fluorescence enhancement as a function of PUMP-DUMP delay. (C). Dependence of STED enhancement of the fluorescence signal on the triplet recovery time In all simulations PUMP and DUMP repetition rates are $76 \mathrm{MHz}$ corresponding to the typical output of modelocked laser systems. 


\section{MATERIALS AND METHODS II - FLUORESCENCE CORRELATION SPECTROSCOPY}

\subsection{Apparatus}

A schematic representation of the single molecule fluorescence system used in this work is shown in figure 6 . The system is based around an inverted Olympus IX-71 microscope with confocal detection of fluorescence excited by a pulsed picosecond fibre coupled amplified diode laser (Picoquant Pico TA) or via a free space port using a continuous wave $405 \mathrm{~nm}$ or $445 \mathrm{~nm}$ laser diode module (Omicron Lasers, Photon Lines). Single molecule fluorescence bursts are detected by two silicon avalanche photodiodes (SPAD, Microphoton Devices SPCM-AQR-14) the output of which are sent to the TCSPC/FCS electronics (Picoquant PicoHarp).

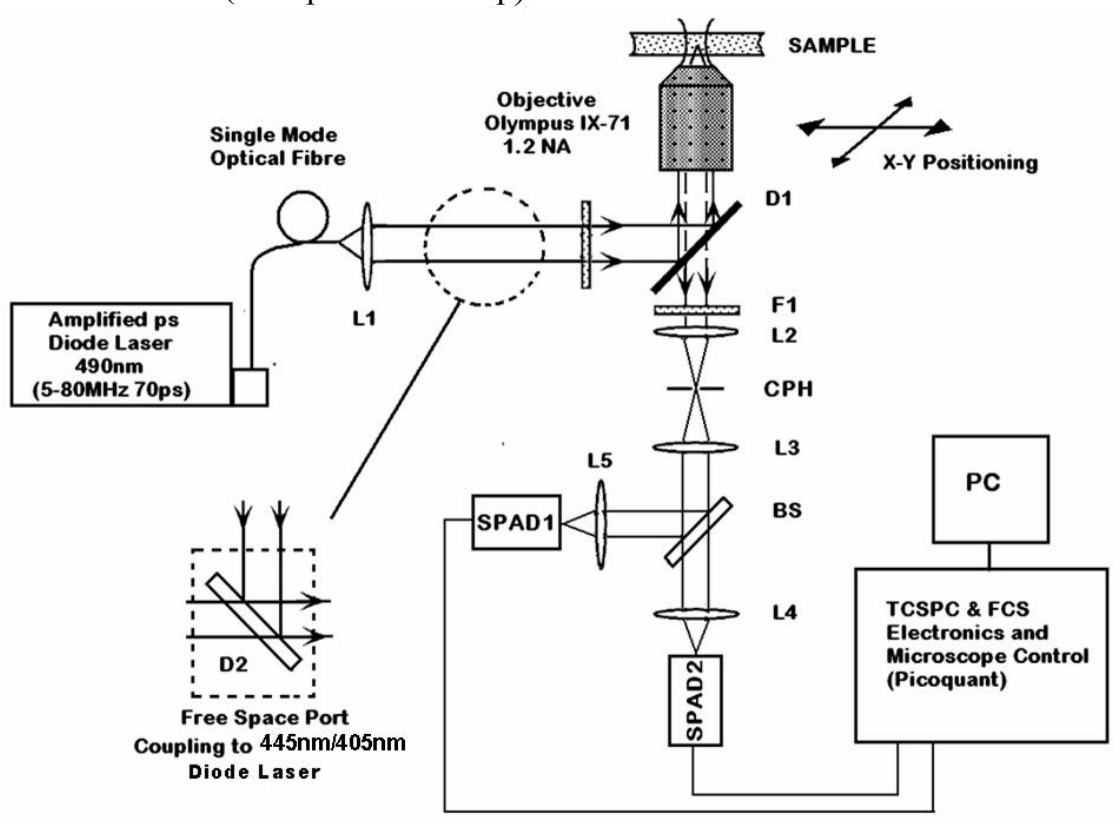

Figure 6. Schematic of the single molecule fluorescence system - fluorescence is detected using a confocal excitation-detection geometry yielding a sub femtolitre volume $\left(0.1-0.5 \times 10^{-15} 1\right.$ depending on the solvent). L2, L3 are confocal focusing and collimation lenses; CPH confocal $(50 \mu \mathrm{m})$ pinhole; L4 \& L5 SPAD focusing lenses; D1 dichroic mirror (505nm long pass); F1 (510nm long pass filter).

\subsection{Calibration of the FCS System}

FCS utilises the temporal fluctuations in the fluorescence emission of small ensembles of molecules in an open excitation volume (sub-femtolitre) that is defined by the confocal geometry of the microscope (figure 6). Analysis of the fluorescence fluctuation autocorrelation $\mathrm{G}^{(2)}(\tau)$ yields information on molecular dynamics in the microsecond to millisecond second time range. The slowest dynamic process to be resolved temporally in a system of mobile molecules is diffusion through the excitation volume. The three-dimensional form of $G^{(2)}(\tau)$ for freely diffusing molecules undergoing faster reversible transitions to a dark state is given by ${ }^{13,14}$,

$G^{(2)}(\tau)=\frac{\langle I(t) I(t+\tau)\rangle}{\langle I(t)\rangle^{2}}=G_{T}^{(2)}(\tau) \times G_{D}^{(2)}(\tau)=\left(1-T_{e q}+T_{e q} \exp \left(-\frac{\tau}{\tau_{T}}\right)\right) /\left[N\left(1+\frac{\tau}{\tau_{D}}\right) \sqrt{1+\frac{\tau \omega_{0}^{2}}{\tau_{D} z_{0}^{2}}}\right]$ 
where $\tau_{\mathrm{D}}$ is the diffusion time of the molecule from the excitation detection volume with radial and axial distances $\omega_{0}$ and $\mathrm{z}_{0}, \mathrm{~N}$ is the (average) number of molecules present and $\tau$ is the time constant associated with the build up of population in the triplet state. $\boldsymbol{T}_{e q}$ is the fraction of dark (triplet states) present and is given by ${ }^{15-17}$

$$
T_{e q}=\frac{k_{23} k_{12}}{k_{12}\left(k_{23}+k_{31}\right)+k_{31}\left(k_{21}+k_{23}\right)}
$$

where $\mathrm{k}_{12}$ is the excitation rate, $\mathrm{k}_{21}$ the spontaneous $\left(\mathrm{S}_{1}\right.$ to $\mathrm{S}_{0}$ decay rate), $\mathrm{k}_{23}$ is the intersystem crossing rate and $\mathrm{k}_{31}$ the triplet recovery rate.

The radial and axial distances $\omega_{0}$ and $z_{0}$ are related to the effective confocal excitation detection volume $V_{\text {eff }}$ by ${ }^{18}$

$$
V_{\text {eff }}=\pi^{3 / 2} \omega_{0}^{2} z_{0}
$$

The radial distance $\omega_{0}$ and axial distance $z_{0}$ was determined from FCS measurements of the translational diffusion of standard fluorophores as outlined below.

\subsection{Determination of FCS parameters in new fluorophores or solvents}

The correlation time for a diffusing species is related to the translational diffusion coefficient by

$$
\tau_{D}=\frac{\omega_{0}}{4 D}
$$

where D is the diffusion coeffiecient of the particle. This coefficient is determined by the Einstein equation for translational diffusion for a given temperature $\mathrm{T}^{19}$ :

$D=\frac{k_{B} T}{6 \pi \eta R_{H}}$

where $k_{B}$ is the Boltzmann constant, $\eta$ is the solvent viscosity and $R_{H}$ is the hydrodynamic radius of the diffusing species. Multiple FCS fits in a standard molecule/solvent environment (that is, a system where the diffusion coeffiecient $\mathrm{D}$ has a published value, such as Rhodamine $6 \mathrm{G}(\mathrm{R} 6 \mathrm{G})$ in water) ${ }^{20}$ can then yield the effective volume (and hence $\omega_{0}$ and $z_{0}$ ) for that solvent. Determination of these parameters in new solvents for a given molecule (and temperature) could be achieved by utilising equation 5 where the ratio of diffusion coefficients is equal to the reciprocal ratio of solvent viscosities.

Calibration of the microscope for OM77 in methylcyclohexane (MCH) was achieved by FCS measurements in this manner on a series of intermediate probe/solvent ensembles (R6G in water, R6G in methanol, Coumarin 6 in methanol, Coumarin 6 in $\mathrm{MCH}$ ). The diffusion parameters and effective volume for OM77 in $\mathrm{MCH}$ are shown in table 2, with the initial calibration system (Rhodamine $6 \mathrm{G}$ in water) also shown for comparison.

\begin{tabular}{|c|c|c|c|c|c|}
\hline Molecule & Solvent & Concentration $(\mathbf{n M})$ & Veff (fl) & Diffusion Time $(\boldsymbol{\mu s})$ & Diffusion Constant $_{\left(\boldsymbol{\mu m} \mathbf{m}^{2} \mathbf{s}^{\mathbf{1}}\right)}$ \\
\hline R6G & Water & $0.1-10$ & 0.54 & $47 \pm 5$ & $280\left(\mathrm{Standard}^{20}\right.$ \\
\hline OM77 & MCH & $1-30$ & 0.24 & $63 \pm 9$ & $95 \pm 28$ \\
\hline
\end{tabular}

Table 2. Calibration of the confocal volume and diffusion characteristics of OM77 (the standard, Rhodamine 6G in water, is also shown) 


\section{FCS EXCITED STATE PHOTOPHYSICS}

Following calibration of the FCS system power dependent FCS measurements were carried out at $490 \mathrm{~nm}$ using the Pico TA amplified diode laser. Although fluorescent, OM77 is weakly absorbing at $490 \mathrm{~nm}\left(\sigma=8.14 \times 10^{-19} \mathrm{~cm}^{2}\right)$ and no discernable changes in the triplet fraction were observed. Instead, FCS measurements were carried out using $\mathrm{CW}$ excitation at $405 \mathrm{~nm}\left(\sigma=2.65 \times 10^{-16} \mathrm{~cm}^{2}\right)$ and $445 \mathrm{~nm}\left(3.95 \times 10^{-16} \mathrm{~cm}^{2}\right)$. The single photon absorption of OM77 peaks at $427 \mathrm{~nm}^{9}\left(\sigma=4.07 \times 10^{-16} \mathrm{~cm}^{2}\right)$.
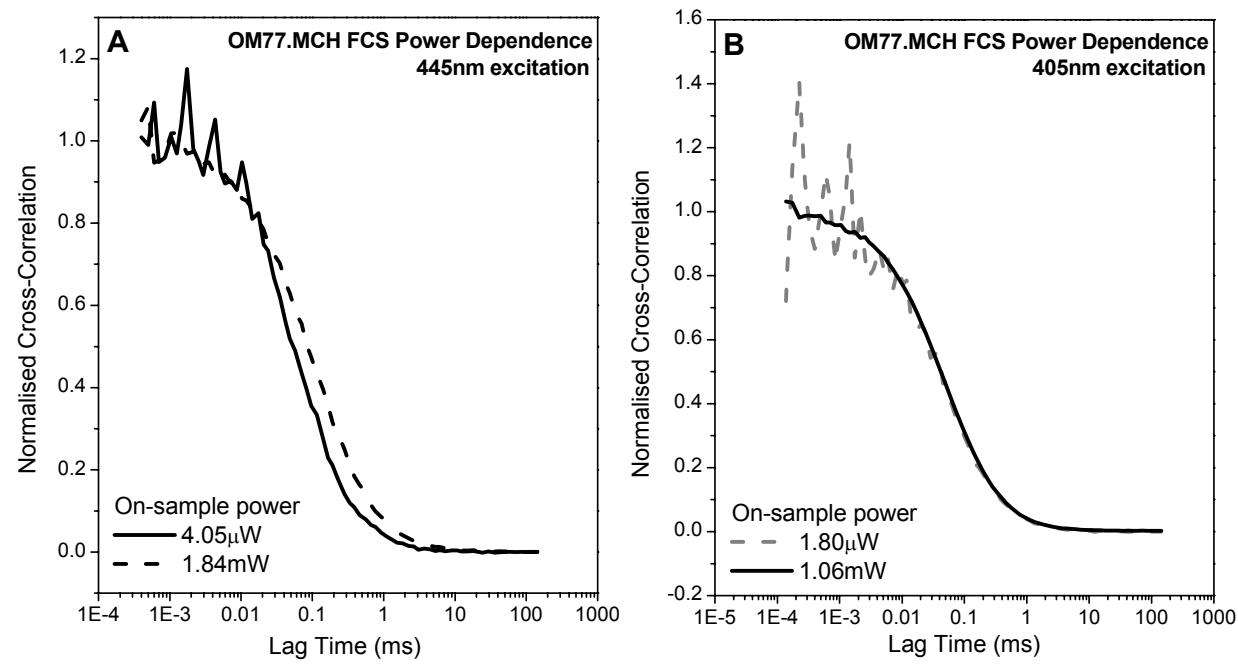

Figure 7. FCS correlation curve variation in OM77 with excitation intensity for two excitation wavelengths ( $445 \mathrm{~nm}$ and $405 \mathrm{~nm})$.
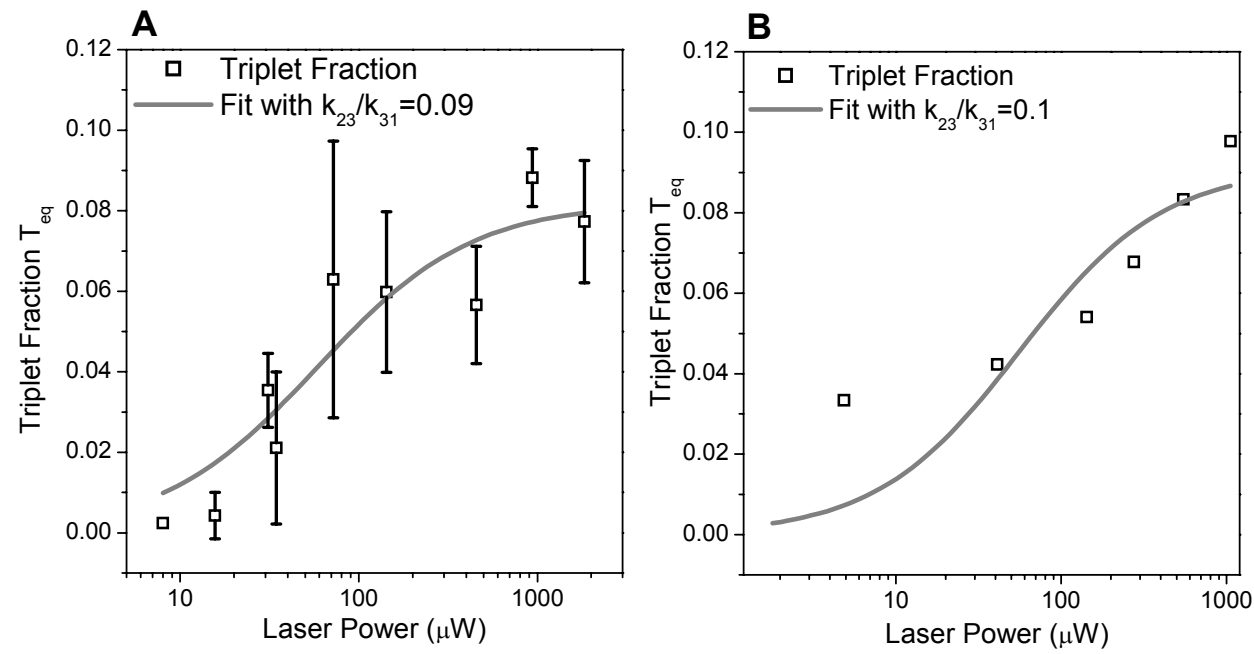

Figure 8. Variation in OM77 triplet fraction with laser power at (A) $445 \mathrm{~nm}$ and (B) $405 \mathrm{~nm}$ excitation. The data is fit to equation (2) assuming a linear dependence of $\mathrm{k}_{12}$ (excitation rate) on average laser power, both moments yield a ratio of $\mathrm{k}_{23} / \mathrm{k}_{31}$ of ca. 0.1 . 
In OM77 a quantum yield of 0.74 and a fluorescence lifetime of $850 \mathrm{ps}$ imply that the radiative and total non-radiative relaxation rates of $S_{1}$ are $8.71 \times 10^{8} \mathrm{~s}^{-1}$ and $3.06 \times 10^{8} \mathrm{~s}^{-1}$ respectively. From figure 8 it can be seen that it was not possible to saturate fully the triplet fraction. Fits of equation 2 to the data assuming a linearity of $\mathrm{k}_{12}$ to $\mathrm{CW}$ excitation power ${ }^{15-17}$ yield ratios of $k_{23} / \mathrm{k}_{31}$ between 0.09 and 0.1 . The absence of strong triplet saturation due to insufficient laser power is consistent with previous measurements of this type, although we are close to the $\mathrm{CW}$ excitation intensities employed by Widengren et $\mathrm{al}^{15}$ to fully saturate the triplet fraction in Rhodamine $6 \mathrm{G}\left(2.8 \times 10^{7} \mathrm{Wcm}^{-2}\right.$ vs $\left.1.33 \times 10^{7} \mathrm{Wcm}^{-2}\right)$.

In non-deoxygenated solutions the triplet relaxation rates are strongly dependent on solvent viscosity and appear to be dominated by oxygen quenching ${ }^{16}$. If we were to infer that the non-radiative relaxation rate of $S_{1}$ was entirely due to intersystem crossing $\left(\mathrm{k}_{23}\right)$ then the triplet decay rate $\mathrm{k}_{31}$ for OM77 is on the order of $3 \times 10^{9} \mathrm{~s}^{-1}-$ a triplet lifetime of $330 \mathrm{ps}$. This is several orders of magnitude shorter than measured (diffusional) triplet times in solvents of similar viscosities ${ }^{16}$ which are typically in the microscecond range. We can therefore conclude that non-radiative relaxation of OM77 is dominated by processes other than intersystem crossing, taking the triplet decay rate to be $10^{6} \mathrm{~s}^{-1}$ this yields an intersystem crossing rate $\mathrm{k}_{23} \approx 10^{5} \mathrm{~s}^{-1}$ which is significantly smaller than the non-radiative relaxation rate of $3.06 \times 10^{8} \mathrm{~s}^{-1}$ that is inferred from lifetime and quantum yield measurements.

It should be noted that the absolute knowledge of $\mathrm{k}_{23}$ and $\mathrm{k}_{31}$ is not crucial in assessing the effectiveness of STED in the reduction of triplet trapping. For OM77 the triplet relaxation rate is an order of magnitude greater than that of intersystem crossing. Under these conditions only a small portion of the 'fluorescence potential' is lost due to triplet trapping as a result of rapid oxygen quenching and the enhancement due to STED will be small.

\section{CONCLUSIONS}

We have shown that STED in single molecules can in principle be achieved in single molecules with current laser technology. Pulsed diode lasers and (pulse stretched) optical parametric oscillators are capable of providing the photon flux needed to strongly drive the DUMP transition (table 1).

STED enhancement of single molecule fluorescence is strongly dependent on excited state relaxation processes. The predominant non-radiative decay in OM77 does not appear to be intersystem crossing. This affects our ability to fully saturate the triplet fraction and accurately determine the absolute values of $k_{23}$ and $k_{31}$. These can in principle be obtained by simultaneous fitting of equations 1 and $2^{16}$ and only becomes possible with significantly higher excitation powers or a substantial decrease in $\mathrm{k}_{31}$. Quenching of the triplet state by oxygen opens up the possibility of photochemical reactions and a reduction in the single molecule lifetime. Triplet relaxation rates in de-oxygenated media are up to three orders of magnitude lower ${ }^{16}$ and under such conditions STED enhancement of single molecule fluorescence should be significant.

\section{ACKNOWLEDGEMENTS}

We wish to thank EPSRC for funding this work.

\section{REFERENCES}

[1] Ishijima, A. and Yanagida T., "Single Molecule Nanobioscience", Trends in Biochem. Sci. 26 (7), 438-444 (2001).

[2] Wölcke, J. and Ullmann, D., "Miniaturized HTS technologies - uHTS", Drug Discovery Today 6 (12), 637-646

(2001).

[3] Rigler, R. and Seela, F., "DNA-Sequencing at the Single Molecule Level”, J. Biotech. 86 (3), 161 (2001).

[4] Vargas, F., Hollricher, O., Marti, O., de Schaetzen, G. and Tarrach, G., "Influence of protective layers on the blinking of fluorescent single molecules observed by confocal microscopy and scanning near field optical microscopy", J. Chem. Phys. 117 (2), 866-871 (2002). 
[5] Balasubramanian, S., Klenerman, D., Barnes, C. L. and Osborne, M. A., PCT patent no. WO00/06770 (10th February 2000).

[6] Osborne, M. A., Barnes, C. L., Balasubramanian, S. and Klenerman, D., "Probing DNA Surface Attachment and Local Environment Using Single Molecule Spectroscopy”, J.Phys. Chem. B. 105 (15), 3120-3126 (2001).

[7] Klar, T. A., Jakobs, S., Dyba, M., Egner, A. and Hell, S. W., "Fluorescence Microscopy with Diffraction Resolution Barrier Broken by Stimulated Emission", Proc. Nat. Acad. Sci. 97 (15), 8206-8210 (2000).

[8] Marsh, R. J., Bain, A. J. and Osborne, M. A., "Control of single molecule fluorescence dynamics by stimulated emission depletion", Proc. SPIE 5222, 78-86 (2003).

[9] Katan, C., Tretiak, S., Werts, M. H. V., Bain, A. J., Marsh, R. J., Leonczek, N., Nicolaou, N., Badaeva, E., Mongin, O. and Blanchard-Desce, M., "Two Photon Transitions in Quadrupolar and Branched Chromophores: Experiment and Theory", J. Phys. Chem. B 111, 9468-9483(2007).

[10] Marsh, R. J., Leonczek, N., Armoogum, D. A., Porres, L., Mongin, O., Blanchard-Desce, M. and Bain, A. J., "Stimulated emission depletion dynamics in push-push polyenes", Proc. SPIE 5510, 117-128 (2004).

[11] Marsh, R. J., Armoogum, D. A. and Bain, A. J., "Stimulated Emission Depletion of Two-Photon Excited States", Chem. Phys. Lett. 366, 398-405 (2002).

[12] Armoogum, D. A., Marsh, R., J. and Bain, A. J., "Polarised Stimulated Emission Depletion of Two-Photon Excited States", Proc SPIE 5222, 34-44 (2004).

[13] Dittrich, P. S. and Schwille, P., "Photobleaching and stabilization of fluorophores used for single-molecule analysis with one- and two-photon excitation", Appl. Phys. B. 73 829-837 (2001).

[14] Schwille, P., Kummer, S., Heikal, A. A., Moerner, W. E. and Webb, W. W., "Fluorescence Correlation Spectroscopy Reveals Fast Optical Excitation-Driven Intramolecular Dynamics of Yellow Fluorescent Proteins", Proc. Nat. Acad. Sci. U.S.A. 97, 151-156 (2000).

[15] Widengren, J., Rigler, R. Mets, U., "Triplet-State Monitoring by Fluorescence Correlation Spectroscopy", J. Fluorescence 4, 255-258 (1994).

[16] Widengren, J., Mets, U. and Rigler, R., "Fluorescence Correlation Spectroscopy of Triplet States in solution: a theoretical and experimental study", J. Phys. Chem. 99, 13368-13379 (1995).

[17] Widengren, J., Mets, U. and Rigler, R., "Photodynamic properties of green fluorescent proteins investigated by fluorescence correlation spectroscopy", Chem. Phys. 250, 171-186 (1999).

[18] Krichevsky, O. and Bonnet, G., "Fluorescence Correlation Spectroscopy: the technique and its applications", Rep. Prog. Phys. 65, 251-297 (2002).

[19] Visser, N. V., Hink, M. A., van Hoek, A. and Visser, A. J. W. G., "Comparison Between Fluorescence Correlation Spectroscopy and Time-Resolved Fluorescence Anisotropy as Illustrated with a Fluorescent Dextran Conjugate", J. Fluorescence 9 (3), 251-255 (1999).

[20] Magde, D., Elson, E. L. and Webb, W. W., "Fluorescence Correlation Spectroscopy. II. An Experimental Realisation”, Biopolymers 13 (1), 29-61 (1974). 